\title{
Progress on Ultra-Wideband (UWB) Multi-Antenna radar imaging for MIGA
}

\author{
Matthew Yedlin ${ }^{1, a}$, Jean-Yves Dauvignac ${ }^{2}$, Nicolas Fortino ${ }^{2}$, Christian Pichot $^{2}$, Raul Cova ${ }^{3}$, \\ David Henley ${ }^{3}$, Kevin Hall ${ }^{3}$, and Stéphane Gaffet ${ }^{4}$ \\ ${ }^{1}$ Department of Electrical and Computer Engineering, University of British Columbia, \\ 2332 Main Mall Vancouver, B.C. V6T 1Z4, Canada \\ ${ }^{2}$ Laboratoire d'Electronique, Antennes et Télécommunications (LEAT) Université Nice - Sophia \\ Antipolis, CNRS Bât. Forum, Campus SophiaTech, 930 Route des Colles, 06903 Sophia Antipolis, \\ France \\ ${ }^{3}$ CREWES (Consortium for Research in Elastic Wave Exploration Seismology) Department of \\ Geoscience University of Calgary, 2332 Main Mall Calgary, Alberta, T2N 1N4, Canada \\ ${ }^{4}$ Low Background Noise Inter-disciplinary Underground Science and Technology, LSBB \\ Underground Research Laboratory, UMS3538 AMU/CNRS/UAPV/UNS La Grande Combe, \\ 84400 Rustrel, France and GEOAZUR, UMR 7329 UNS/CNRS/IRD/OCA, 250 rue Albert Einstein, \\ Bât. 4, Sophia-Antipolis, 06560 Valbonne, France
}

\begin{abstract}
Progress on the development of the multi-channel, ground penetrating radar imaging system is presented from hardware and software perspectives. A new exponentially tapered slot antenna, with an operating bandwidth from $100 \mathrm{MHz}$ to $1.5 \mathrm{GHz}$ was fabricated and tested using the eight-port vector network analyzer, designed by Rhode and Schwarz Incorporated for this imaging project. An eight element antenna array mounted on two carts with automatic motor drive, was designed for optimal common midpoint (CMP) data acquisition. Data acquisition scenarios were tested using the acoustic version of the NORSAR2D seismic raytracing software. This package enables the synthesis and analysis of multi-channel, multi-offset data acquisitions comprising more than a hundred thousand traces. Preliminary processing is in good agreement with published bistatic ground-penetrating radar images obtained in the tunnels of the Low-noise Underground Laboratory (LSBB) at Rustrel, France.
\end{abstract}

\section{Introduction}

The universities of Avignon, Nice Sophia Antipolis, British Columbia and Calgary are constructing a multi-channel ground-penetrating radar(gpr) imaging system to support the Matter-wave laser Interferometric Gravitation Antenna (MIGA) project. This system is based on ANR Maxwell, a prototype funded by the ANR. Our research focuses on the design and construction of an 8 element ultra-wideband antenna array. Preliminary raytracing models of data to be collected by this new system match the images obtained by Sénéchal et al. [1].

\footnotetext{
${ }^{\mathrm{a}}$ Corresponding author: matty@ece.ubc.ca
} 


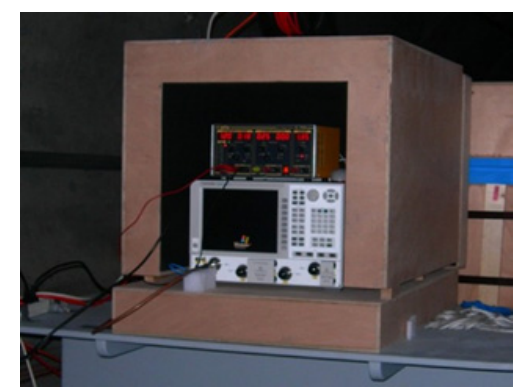

a)

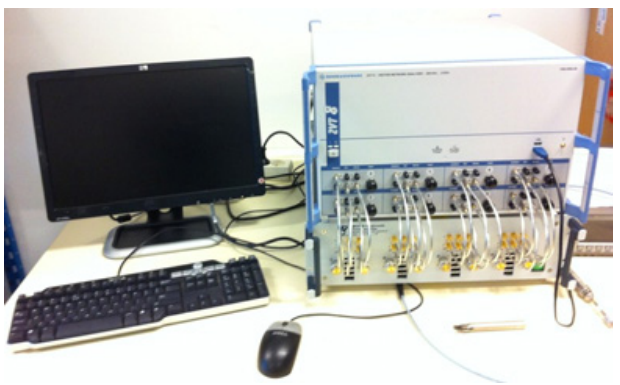

b)

Figure 1. a) ANR MAXWELL VNA. b) Project MIGA Rhode and Schwarz VNA.

\section{Imaging system progress - hardware}

\subsection{Antenna development}

The proposed ultra-wideband (UWB) microwave imaging system consists of a customized Vector Network Analyzer (VNA) connected to eight specifically designed antennas. These antennas are integrated in an autonomous shielded carriage. The previous ANR MAXWELL gpr imaging project $[2,3]$ used an external amplifier as shown in Fig. 1a. The new frequency range of this UWB ground-penetrating radar extends from $100 \mathrm{MHz}$ to $1.5 \mathrm{GHz}$ to obtain a good trade-off between penetration depth, spatial resolution, and antenna size. This UWB system is designed to provide fast, multi-static, multipolarization, wide offset measurement capabilities with a high dynamic range and a better timing resolution than narrow band systems.

A large dynamic range, 8-port VNA, shown in Fig. 1b, connected to additional customized amplifiers, is used for recording the reflected electromagnetic waves received by the antennas. Our equipment offers high dynamic range measurement (typically $130 \mathrm{~dB}$ ) to monitor the water content of the unsaturated area of the Vaucluse Karst aquifer under the LSBB site, at decametric depth. This new acquisition system includes 8 high gain integrated amplifiers that are specifically shielded to avoid spurious radiation which was noticeable in the previous MAXWELL system.

A new ultra wide band printed antenna was specifically designed to fit the project requirements [4, 5]. Classical Tapered Slot Antennas (TSA) have a low profile, a wide bandwidth, and superior radiation characteristics. However, the TSA size limits their integration capabilities in the antenna array carriage (size must be kept below $75 \mathrm{~cm}$, which is $\lambda / 4$ at $100 \mathrm{MHz}$ compared to the typical $\lambda / 2$ ). We used new loading techniques to place the CMS resistive loads to achieve this design goal without modifying antenna performance. Insertion of loads, commonly used in radar applications, increases antenna bandwidth and minimizes return losses. This technique, however, usually degrades antenna efficiency and as a result, its gain. For the proposed antennas, the placement of loads was carefully studied to extend bandwidth to lower frequencies without affecting antenna radiation at higher frequencies. Moreover, this modification increases antenna directivity. The advantages of having a higher directivity are twofold: a higher gain at low frequencies (the lack of efficiency is over-compensated) and a lower coupling between neighboring antennas in the array. This novel ETSA, shown in Fig. 2, demonstrates excellent impedance matching from $80 \mathrm{MHz}$ to $2 \mathrm{GHz}$, a maximum gain of $9 \mathrm{~dB}$, and an improved impulse response. 


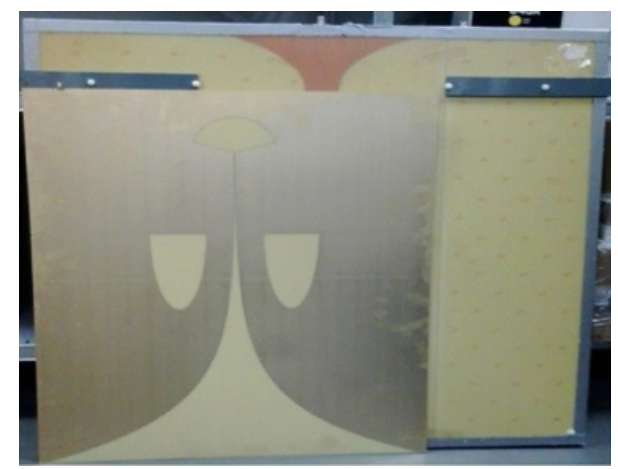

Figure 2. New miniaturized ETSA (Exponentially Tapered Slot Antenna) superimposed on original larger ETSA.The exponential taper (a horn-shape) is clearly visible at the bottom of the figure.

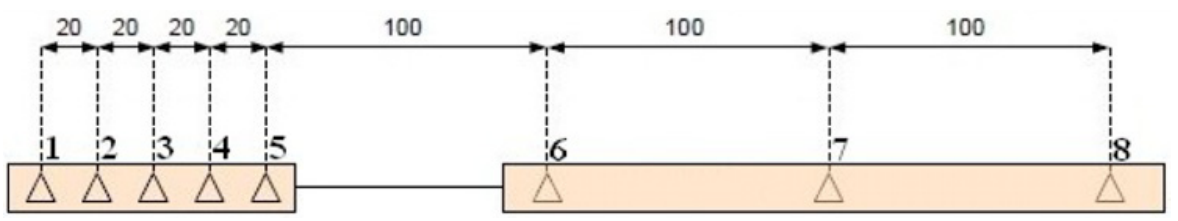

Figure 3. Schematic of antenna array with 20 and $100 \mathrm{~cm}$ antenna element spacing.

\subsection{Antenna array}

The array is separated in two carts linked together, illustrated schematically in Fig. 3. The first one is motorized and provides the traction of the array. It embeds an array of 5 shielded antennas separated by $20 \mathrm{~cm}$. The second one is placed so that its first antenna is separated by $1 \mathrm{~m}$ from the last antenna of the tractor cart. It embeds an array of 3 shielded antennas separated by $1 \mathrm{~m}$. Using this array geometry, the auscultation of a $200 \mathrm{~m}$ gallery with a $10 \mathrm{~cm}$ step generates 2500 frequency measurements for each position and each antenna transmission between the 8 antennas. The amount of collected data exceeds 19 classical CMP runs on the same zone and takes less than 17 hours with an ultra-wide bandwidth measurement. The flexibility of the instrument also offers the possibility to prioritize a fast sweep (time measurement divided by 7 for a $120 \mathrm{~dB}$ dynamic range) for a simple survey, or a high dynamic range (up to $140 \mathrm{~dB}$ ) for further investigations focused on a specific zone.

\section{Imaging system progress - synthetic image simulation at LSBB}

\subsection{Theory}

This data is generated using the NORSAR seismic two-dimensional ray tracing software, in an acoustic setting. After simple linear scaling, the acoustic wave equation can be used to model TE-polarized electromagnetic waves as shown by Laurain and Lecomte [6]. They demonstrate clearly that the TE reflection coefficients correspond precisely to the acoustic reflection coefficients with appropriate conversions of impedance and velocity. For this study, 


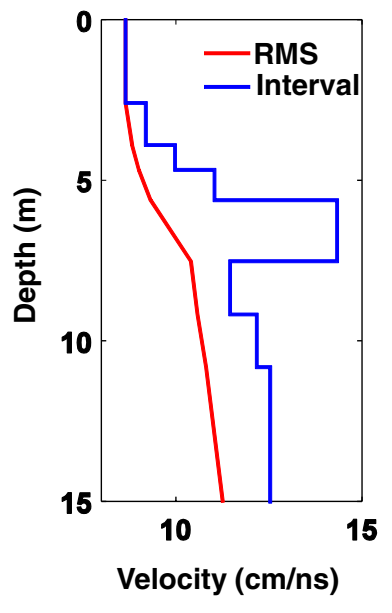

a)

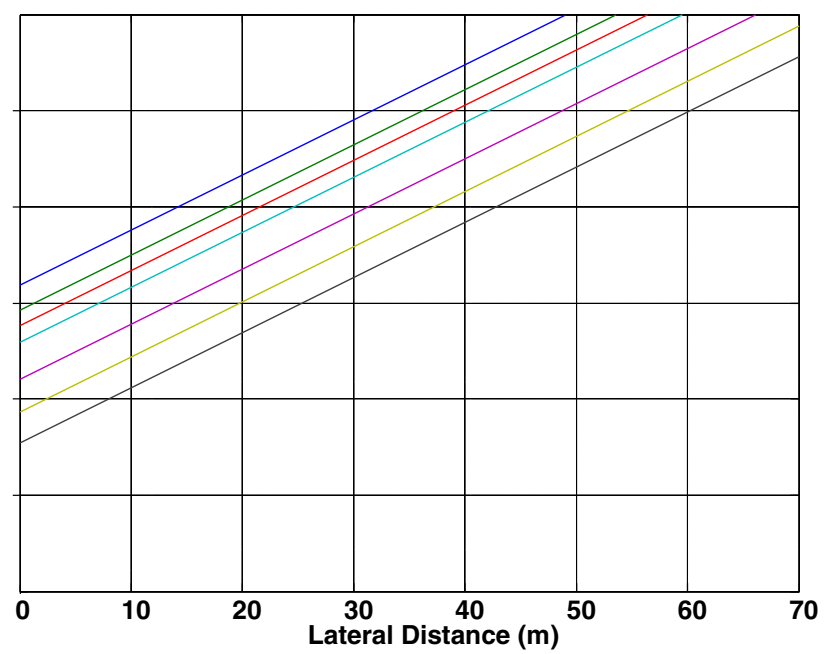

b)

Figure 4. a) RMS and interval velocities obtained from Fig. 5a in [1]. b) Interpolated velocity model obtained from Fig. 4 in [1].

primary reflections, from layer boundaries that enclose constant parameter layers, have been utilized.

\subsection{Case study}

We have chosen Figs. 4, 5a and 5b from the 2013 paper by Sénéchal et al. [1] to create synthetic data of the type that would be collected by the antenna array shown in Fig. 3. Figure 4 was used to create the velocity structure model. Figures $5 \mathrm{a}$ and $5 \mathrm{~b}$ were used to create the RMS and interval velocities.

Shown in Fig. 4, panel a) are the RMS velocities that were picked from the Fig. 5b in [1]. The interval velocities, shown in panel a) were obtained using the Dix equations [7], with a dip correction corresponding to a dip of 16 degrees. In Fig. 4, panel b), the structural model, corresponding to Fig. 4 in [1] is presented. The dipping layers were obtained by linear interpolation. A synthetic CMP gather, without any additive noise, at location $40 \mathrm{~m}$, is presented in panel a) of Fig. 5, with associated semblance in panel b). For comparison, the CMP gather from Fig. 5a and the semblance in Fig. 5b of Sénéchal et al. [1] are presented in panels c) and d) respectively. There is very good agreement of the primary arrival hyperbolae and associated semblance panels. For example, at $60 \mathrm{~ns}$, the hyperbolic caps of the synthetic and real data line up as do the maxima of the semblance panels b) and d). The alignment with the other six hyperbolae shown gives us confidence that our model-building procedure and subsequent ray tracing is adequate to model results in the future.

To validate our proposed modeling of acquired data, in Fig. 6 we present a synthetic source gather in panel a) and the complete CMP stack in panel b). The data in panel a) was created by adding a pseudo-random noise generated from a normal distribution with zero mean, resulting in an SNR of 1.3. It was computed at a source position of the antenna array at location $38 \mathrm{~m}$, near $40 \mathrm{~m}$, the location of the CMP used in the velocity semblance analysis. In panel b) we present the final stacked data, reflecting the original model shown in Fig. 4b. 


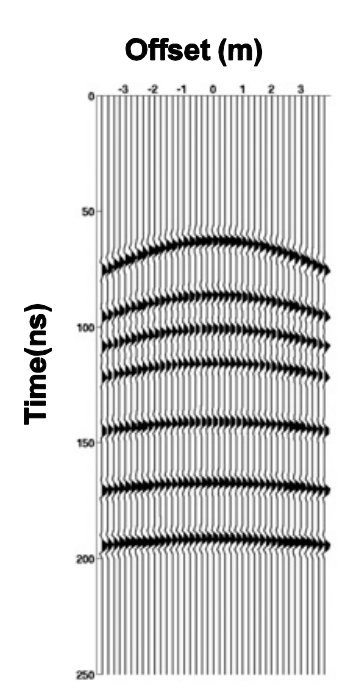

a)

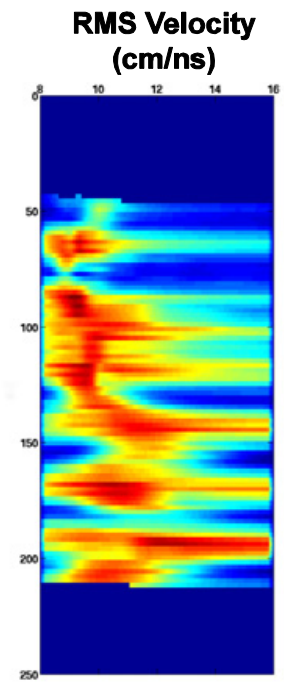

b)

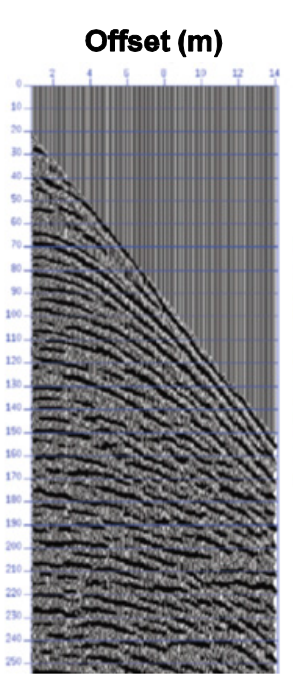

c)

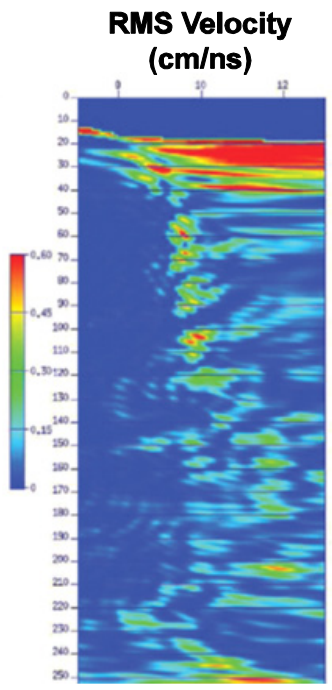

d)

Figure 5. a) Synthetic CMP gather. b) Velocity Semblance computed from a). c) CMP recorded at LSBB [1]. d)Velocity Semblance computed from d).

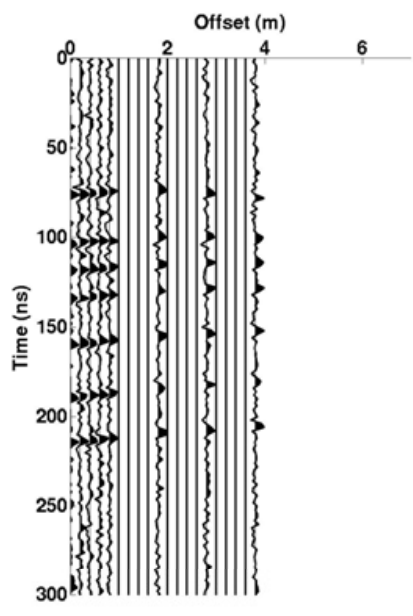

a)

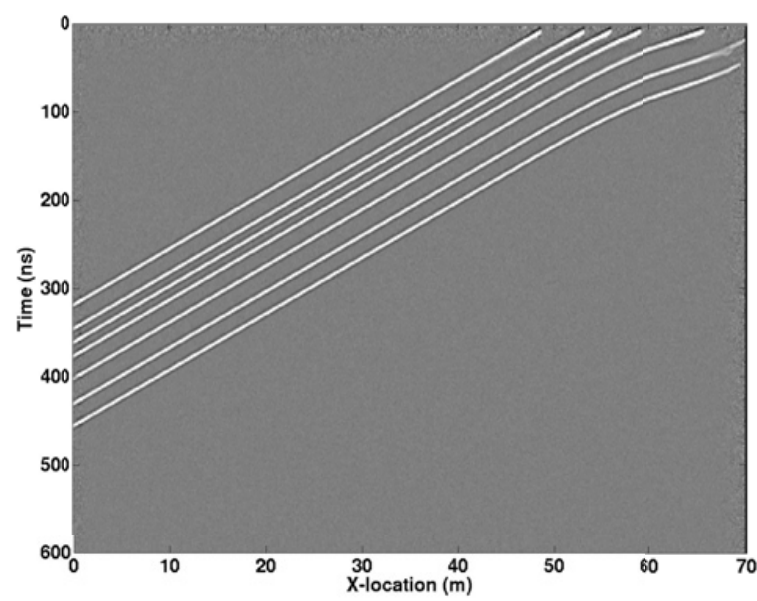

b)

Figure 6. a) Source gather at $38 \mathrm{~m}$. b) Complete stacked section for velocity model.

\section{Conclusions}

In our research paper, we have presented the current progress in the development of an 8 element ground penetrating radar antenna array composed of novel, compact ultra-wideband antennas. Data acquisition is achieved by using the Rhode Schwarz Incorporated ZVT vector network analyzer, with an attached eight channel shielded amplifier, designed specifically for this radar imaging project. 
Concurrent progress in the development of a data simulation platform was demonstrated by using the NORSAR2D ray-tracing software to simulate data that will be recorded at LSBB. Preliminary results demonstrate the viability of the ray-tracing approach necessary to model the 128,000 traces that will be collected at LSBB. This modelling is a necessary first step in creating an inversion algorithm for the determination of subsurface permittivity.

The authors would like to acknowledge support from the ANR(France), NSERC(Canada), CREWES (Consortium at the University of Calgary) and CREWES sponsors.

\section{References}

[1] G. Sénéchal, D. Rousset, S. Gaffet, Near Surface Geophysics 11, 283 (2013).

[2] J. Dauvignac, N. Fortino, G. Sénéchal, A. Cresp, M. Yedlin, S. Gaffet, D. Rousset, and C. Pichot, Ultra-wideband GPR imaging of the Vaucluse Karst Aquifer. In AGU Fall Meeting Abstracts (2008), Vol. 1, p. 08.

[3] M. Yedlin, G. Sénéchal, D. Rousset, N. Fortino, J.Y. Dauvignac, S. Gaffet, T. Monfret, and C. Pichot, Comparative study using an UWB measurement system and a RAMAC GPR system for subsurface imaging of the vaucluse karst aquifer, in Wireless Information Technology and Systems (ICWITS), 2010 IEEE International Conference on (IEEE, 2010), pp. 1-4.

[4] C. Diakité, N. Fortino, J.-Y. Dauvignac. Antenne ETSA "Exponential Tapered Slot Antenna" miniature pour radar à pénétration de surface, in $19^{e ̀ m e s}$ Journées Nationales Microondes (JNM 2015) pp. Session-Antennes.

[5] G. Clementi, N. Fortino, J. Dauvignac, A novel low profile Tapered Slot Antenna with absorbing material for radar imaging system. in Antennas and Propagation (EuCAP), 2013 7th European Conference on (IEEE, 2013), pp. 2891-2895.

[6] R. Laurain, I. Lecomte, Elastic/Electromagnetic Wave Propagation-Equivalences and 2D Modelling of GPR, In 63rd EAGE Conference \& Exhibition (2001).

[7] Ö. Yilmaz, Seismic data analysis, Vol. 1 (Society of exploration geophysicists Tulsa, 2001). 